\title{
Linked Data-based Conceptual Modelling for Recommendation: a FCA-based approach
}

\author{
A. Castellanos, A. García-Serrano, J.Cigarrán \\ ETSI Informática \\ Universidad Nacional de educación a Distancia (UNED) \\ C/ Juan del Rosal 16, 28040 Madrid, Spain \\ \{acastellanos, agarcia, juanci\}@lsi.uned.es
}

\begin{abstract}
In a recommendation task it is crucial to have an accurate contentbased description of the users and the items consumed by them. Linked Open Data (LOD) has been demonstrated as one of the best ways of obtaining this kind of content, given its huge amount of structured information. The main question is to know how useful the LOD information is in inferring user preferences and how to obtain it. In this context, we propose a novel approach for Content Modelling and Recommendation based on Formal Concept Analysis (FCA). The approach is based in the modelling of the user and content related information, enriched with Linked Open Data, and in a new algorithm, to analyze the models and recommend new content. The framework provided by the ESWC 2014 Recommendation Challenge is used for the evaluation of the proposal. The results are within the average range of other participants, so the suitability of FCA for this scenario seems to be addressed. Nevertheless, further work has to be carried out in order to propose a refined approach for the management of LOD information.
\end{abstract}

Keywords: Linked Open Data, Recommender Systems, Content Modelling, Formal Concept Analysis.

\section{Introduction}

Broadly speaking, Recommender Systems (RS) pursue the discovery of new and relevant content. RS has traditionally been addressed by means of two main approaches: Collaborative Filtering (CF) and Content-Based (CB). CF approaches puts users with similar previous behaviours in the same set together (neighbourhood). Then, given a target user, the items consumed by other users in its neighbourhood are considered as suitable recommendations. On the other hand, CB systems base their operation on putting together items with similar content. The recommendation is made by offering similar items to those already consumed by a target user.

A recommendation task has to cope with different problems [1,2] and involves several areas of knowledge, such as personalization [3], information retrieval [4, 5], machine learning [6] or user modelling [7]. The main problems are related to the paucity 
of information (e.g. cold start [8], lack of diversity [9], scarcity [10] or limited recommendation coverage).

Linked Data is shown as a remarkable alternative to solving some of these main problems. Linked Data refers to the set of good practices for publishing, connecting and sharing structured data [11]. Several sources offer linked data through public endpoints (i.e. the so-called Linked Open Data, LOD). One of the most well-known LOD sources is DBPedia ${ }^{1}$, a database which offers Wikipedia data in a structured way. LOD facilitates the acquisition of structured data, suitable to be used for reducing the information scarcity problem in RS. In works [12, 13, 14], LOD has been consumed mainly for data enrichment in order to improve the performance of the recommender systems. However, given a recommendation scenario (user set, items set and the interactions between them), the way in which this data can be linked with the items and users has to be defined. In this sense, some interesting approaches had been looked into, not only in the recommendation context $[15,16,17]$. Basically, these proposals follow two methods: the use of the SPARQL language to search for specific information (i.e. information about a given content or entity) or to take advantage of the LOD structure to infer relationships and obtain related information.

As a result of works such as the aforementioned and other initiatives, several specific datasets have been published. Some interesting include: The DBbook database ${ }^{2}$, The DBPedia mappings to MovieLens1M dataset or DBPedia Mappings to Last.fm dataset $^{3}$.This dataset includes not only the recommendation-based data but also links between the data and LOD sources (mainly DBPedia). Some other efforts stand out, such as the organization of Workshops and Challenges focused on the LOD-based recommendation. For instance, the ESWC-2014 Challenge: Linked Open Data-enabled Recommender Systems, which proposes an evaluation framework for RS experimentation because, as the organizers propose, the application of LOD in RS can lead to the creation of a new breed of knowledge-enabled and content-based RS.

In order to achieve this goal, this paper proposes a novel approach based on Formal Concept Analysis (FCA) [18, 19] for content and user profile modelling. LOD, and more specifically DBPedia-extracted data, are used to create a semantic item description to be taken into account in the modelling step. Based on this FCA modelling, an algorithm is proposed to manage the models and recommend new contents.

FCA has been demonstrated in the past as a powerful technique to organize content. For this reason, previous works have tried to take advantage of this organization performance in the recommendation scenario. FCA has been applied both for CF [20, $21,22]$ and CB approaches [23, 24]. However, most of these works barely delve into the possibilities of FCA for modelling. In this sense, this work, applies some of the conclusions obtained from the aforementioned researches, and tries to go a step further in the application of FCA in a recommendation scenario. More specifically, this work

\footnotetext{
${ }^{1}$ http://dbpedia.org/

${ }^{2} \mathrm{http}$ ://challenges.2014.eswc-conferences.org/index.php/RecSys

${ }^{3} \mathrm{http} / / /$ sisinflab.poliba.it/semanticweb/lod/recsys/datasets/
} 
aims to answer the following question: Can the inferred semantic-based formal concepts be useful in inferring user preferences?

To answer this question, this paper presents an experimental work based on the ESWC 2014 Challenge, through the development of a framework to experiment and evaluate recommendation approaches using LOD. The experimentation is conducted by modelling the LOD information related to the items to be recommended through FCA. The main goal is to prove whether this kind of information can be useful in generating a conceptual based modelling which can link with the user preferences. Our hypothesis is that, if the user preferences (and the subsequent user activity) are somehow dependant on "a relationship" with the content of the items consumed, the power of FCA to organize and model content should be useful in modelling this relationship.

The proposal, the experiments conducted by applying this proposal and finally the obtained results and their analysis is presented below.

\section{Recommendation Approach Description}

The recommendation approach runs on an FCA-based content modelling. By means of FCA an offline model of the data is created. Then, the FCA-based recommendation algorithm explores the offline model to extract suitable relationships containing valuable recommendations. Finally, a pruning of the raw recommendations is conducted to select only the most suitable ones.

\subsection{FCA at a Glance}

FCA is a mathematical theory of concept formation derived from lattice and ordered set theories. FCA provides a theoretical model to organize information and discover relationships $[18,19]$.

The main constructor of the theory is the formal concept that is derived from a formal context. A formal context $\mathbb{K}:=(G, M, I)$, can be seen as an incidence matrix that presents the set of has- $a$ relationships $(I)$ between objects $(G$, from the German word Gegenstände) and attributes ( $M$, from the German Merkmale). An example of a formal context is shown in Fig. 1(a).

Based on the information contained in the formal context, the set of formal concepts can be generated. Strictly, a formal concept is a pair $(A, B)$, where $A \subseteq G$ is a set of objects (also known as the extent of the formal concept), and $B \subseteq M$ is a set of attributes (also known as the intent of the formal concept), such as:

- The extent $A$ consists of all the objects that are related to all the attributes in the intent $B$.

- The intent $B$ consists of all the attributes shared by the objects in extent $A$.

Formal concepts can be ordered by their extents in a subconcept-superconcept relationship according to their extents:

$$
(A, \mathrm{~B}) \leq(C, \mathrm{D}): \Leftrightarrow(\mathrm{A}, \mathrm{B}) \subseteq(\mathrm{C}, \mathrm{D}) \Leftrightarrow \mathrm{A} \subseteq \mathrm{C}
$$


In this case, $(C, \mathrm{D})$ is called a super-concept of $(A, \mathrm{~B})$ and, conversely, $(A, \mathrm{~B})$ is a sub-concept of $(C, \mathrm{D})$. This ordered relationship is a generalization-specialization, and it can be proven to be a lattice (i.e., a concept lattice denoted as $\mathcal{B}(G, M, I)$,) based on the basic FCA theorem [18, 19].

(a)

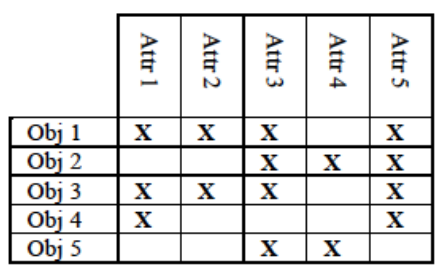

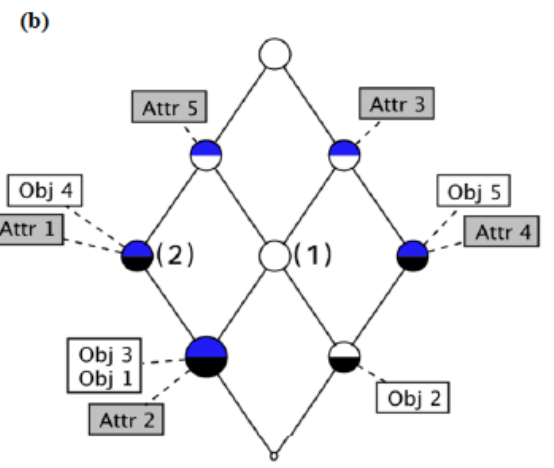

Fig. 1. (a) A sample formal context and (b) the concept lattice associated with the formal context in (a)

In a concept lattice, two important types of formal concepts are the object concepts and the attribute concepts:

- The object concept, denoted as $\gamma g$, associated with an object $g$ is the most specific concept that includes $g$ in its extent. The intent of an object concept is defined by all the attributes of $g$, whereas the extent contains not only object $g$ but also all those objects related to all the attributes of $g$.

- The attribute concept, denoted as $\gamma m$, associated with attribute $m$ is the most generic concept including $m$ in its intent. Its extent contains all the objects with attribute $m$, and its intent is defined by all the attributes shared by the objects belonging to the extent set.

Because concept lattices are ordered sets, they can be naturally displayed in terms of Hasse diagrams. In a Hasse diagram: a) there is exactly one node for each formal concept; b) if, for concepts $C 1$ and $C 2, C 1 \subseteq C 2$ holds, then $C 2$ is placed above $C 1$; and c) if $C 1 \subseteq C 2$ but there is no other concept $C 3$ so that $C 1 \subseteq C 3 \subseteq C 2$, there is a line joining $C 1$ and $C 2$. Fig. 1a shows an example of a formal context, and Fig. 1b shows its associated concept lattice using a Hasse diagram.

\subsection{FCA-based Content Modelling}

In the previous section the basis of FCA has been outlined. To sum up, any data that can be represented as a formal context is susceptible to being modelled by applying FCA. In this sense, recommendation context can be interpreted as a bipartite graph 
partitioned into users $(U)$ and items $(I)$. The edges in this graph, $\rho=r(u, i)$, establish the relationship of the user $u$ with an item $i$ weighted with a rating $r$.

Following the FCA theory, the triple (U, I, $\rho$ ) can be interpreted as a formal context (or a recommendation context). From this recommendation context, a set of formal concepts $(A, \mathrm{~B})$ can be inferred, where $\mathrm{A}$ is the user set sharing the item set $\mathrm{B}$ (i.e. users in A liked/consumed/positively rated items in B). By applying the subconceptsuperconcept relationship, the set of formal concepts can be ordered in a recommendation lattice, which can be represented according to a Hasse Diagram. There are going to be several formal concepts including users with the same previous behaviour, from the more generic to the more specific. This user-item modelling is the so-called UserItemLattice. The UserItemLattice reflects a CF recommendation scenario: users are organized according to their shared items. Nevertheless, FCA can be also applied to the CB scenario: organizing items according to their content. In this case, the recommendation context includes the relationships between items and their content that are the LOD-based features. Consequently, the set of inferred formal concepts $(I, \mathrm{C})$ will include the items (C) sharing a set of content $(C)$. The recommendation lattice that organizes these formal concepts will be referred to as the ItemContentsLattice.

\subsection{Recommendation Approach}

The recommendation approach proposed is quite similar to that proposed in [25], to take advantage of the structure of the concept lattice to find suitable recommendations. Two recommendation approaches are proposed: A CF approach based on the UserItemLattice and a $\mathrm{CB}$ approach based on the ItemContentsLattice. The CF approach extracts the recommendations by applying the following algorithm to each user $u$ to be recommended:

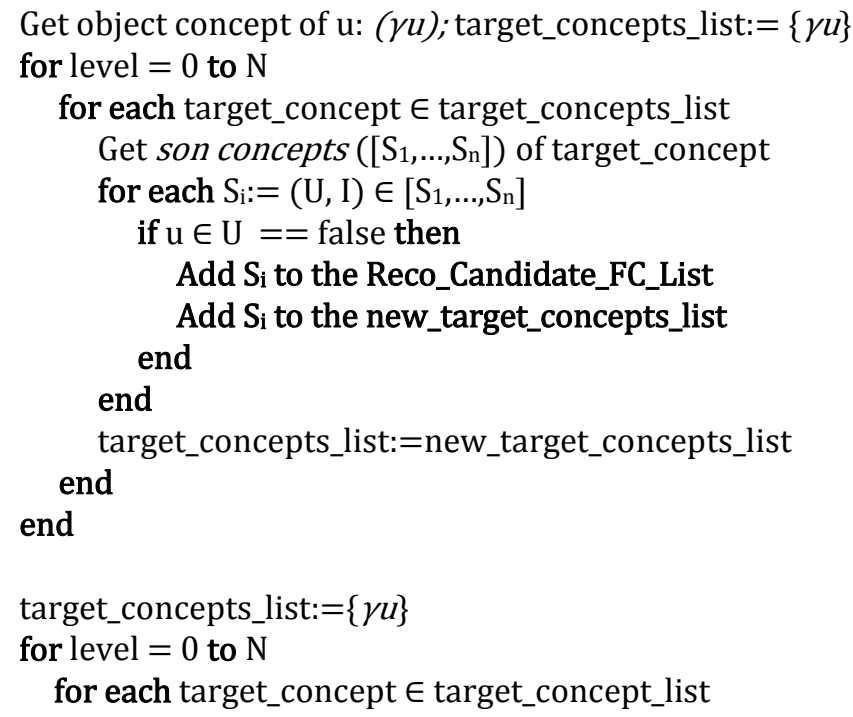




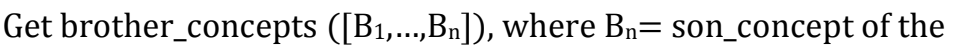
parent_concept

for each $B_{i}:=(U . I) \in\left[B_{1}, \ldots B_{n}\right]$

if $u \in U==$ false then

Add $B_{i}$ to the Reco_Candidate_FC_List

Add $B_{i}$ to the new_target_concepts_list end

end

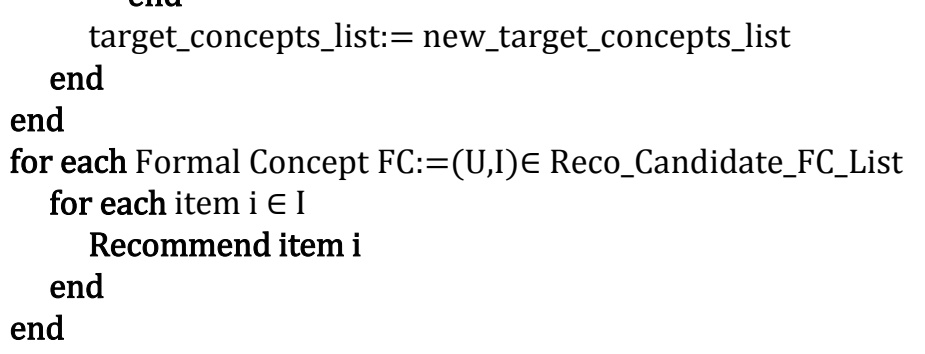

Fig. 2. Collaborative Filtering based Recommendation Algorithm. Being $N$ the number of levels (above and below the target concept level) to look for recommendations, a son concept the concept linked in the level below of a given one and a parent concept the concept linked in the level above of a given one.

The $\mathrm{CB}$ algorithm is also based on the lattice structure to conduct the recommendations, although its functioning is slightly different. Given a user to be recommended and its item set (i.e. the items consumed by them), the recommendations are offered by apply the following algorithm:

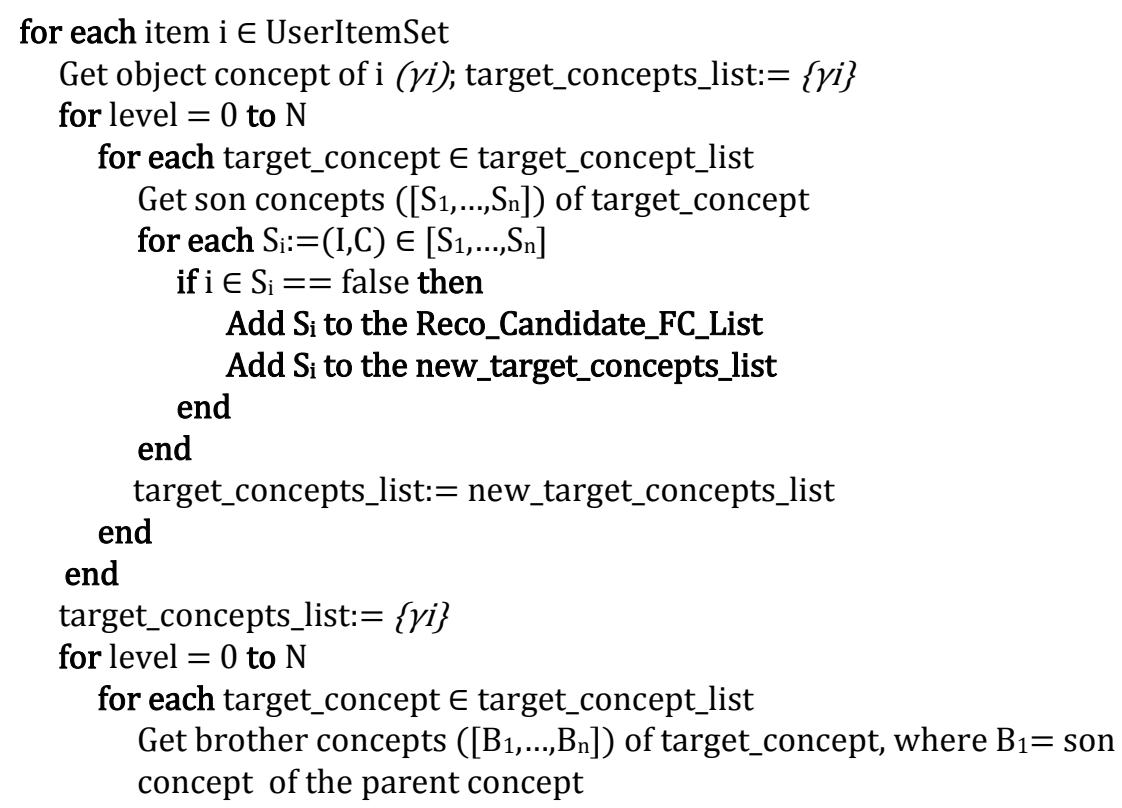




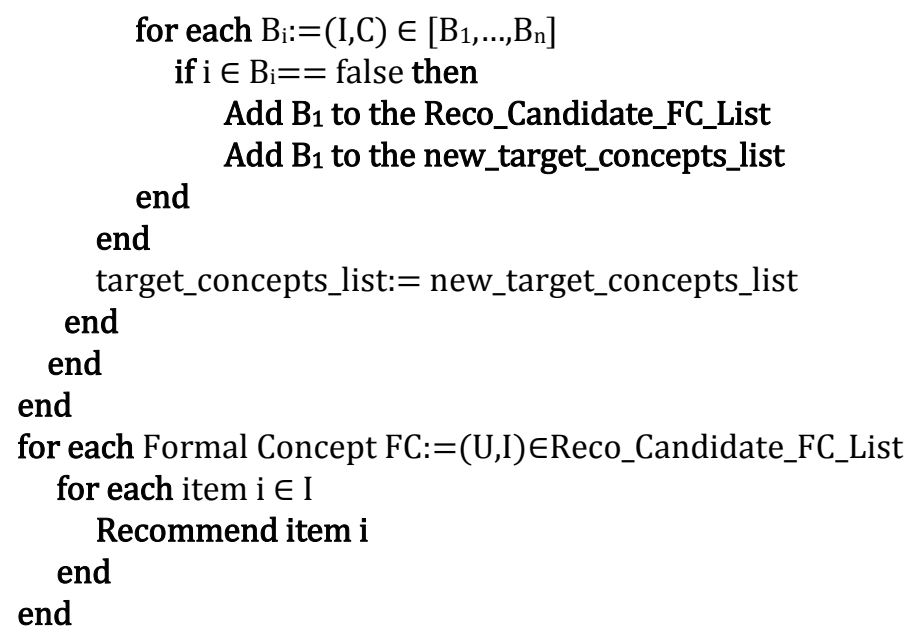

Fig. 3. Content Based Recommendation Algorithm. Being N the number of levels (above and below the target concept level) to look for recommendations, a son concept the concept linked in the level below of a given one and a parent concept the concept linked in the level above of a given one.

\section{Performed Experiments}

The test bed ESWC 2014 Recommendation Challenge ${ }^{4}$, and the experiment performed as well as the results obtained and its comparison with the results of the rest of participants is presented below.

\subsection{The ESWC 2014 Recommendation Challenge}

The ESWC 2014 Recommendation Challenge pursues the experimentation through the application of LOD to the recommendation task. To facilitate the participation, the organizers provided the participants with the DBbook dataset and with the definition of three tasks:

- $\quad$ Task 1: Predict Missing Ratings

- Task 2: Order an item set according to a predicted recommendation score

- Task 3: Generate a Top-20 Recommendation List

The DBbook dataset is made up of (a) more than 70,000 interactions between users, (b) an item set made up of more than 8,000 books and (c) the DBPedia endpoint of each item in the collection.

The DBbook dataset did not include any kind of content data of the items (i.e. it only had the identifier of the books), but to enrich the annotation of the items, it was possible to take advantage of the DBPedia endpoints and download the information related to them. Nevertheless, the information in DBPedia as regards each item is extensive and

${ }^{4}$ http://challenges.2014.eswc-conferences.org/index.php/RecSys 
related to different aspects. After reviewing all of this information, and taking into consideration the type of item in the dataset (books), we selected only those data we considered more related to the interest of a given user to a given item.

The data selected to enrich the DBbook dataset was: abstract, literaryGenre, country, language, name and subject. Restricting the enrichment process to only DBPedia data does not take advantage of the full power of the LOD Cloud. However, this issue is out of the scope of the main aim of the proposed approach and experiments: to show the advantages of a conceptual data modelling FCA-based applied to a semantic driven representation, no matter what this representation could be. A more sophisticated LODbased enrichment could result in a better general performance (at least theoretically), but it remains as a future work. An interesting reference on how to explore the LOD Cloud and extract information, with many possible applications in recommendation, is presented in [26].

The recommendation approach to perform a "Recommendation in the Wild" is described below

\subsection{Recommendation in the Wild}

This recommendation task is based on predicting the ratings of a group of fixed items so the $\mathrm{CB}$ approach appears to be more suitable than the CF. Thus, the CB approach based on the ItemContentLattice modelling plus the CB-based algorithm was applied. The main goal is to know how the semantic information used in a FCA-modelling can be used in the recommendation problem. The results obtained by the participating groups (our work is identified as UNED group) are shown in Fig. 4 (in the horizontal axe), sorted by the F-Measure value (range from 0,005 to 0,050). The low results obtained by the different groups' shows the difficulty of the task.

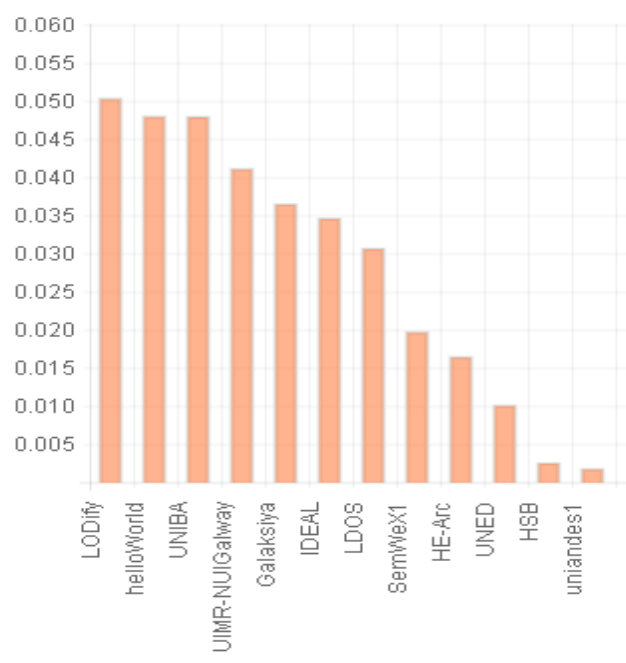

Fig. 4. ESWC2014 Recommendation in the Wild Results 
At this point, this low performance can be ascribed to three causes. The first one, FCA is not suitable if not for recommendation in general, at least for this given task. The second one, the LOD information, although provides an accurate representation of the item content it is not useful in creating a representation that links with the user preferences. Finally, these low results could be only attributed to the high complexity of the task, so that, even though the results were low the proposal would still be valid, The latter is hardly demonstrable; however, we can remove the dependence between the low results and the FCA performance, by applying a "bare" FCA based approach to a different recommendation task (the interest prediction) in order to test its performance.

\subsection{Interest Prediction}

In this task, given an item set to be recommended, it has to be sorted according to a predicted score, related to the degree of interest of a given item for a given user. In the ESWC2104 Challenge, this scenario is handled by tasks 1 and 2, although we focused our experimentation only on task 2 just in order to test how the FCA-based approach performs. The use of the previous user behaviours is the best approach to address this task; that is, the application of a CF system to recommend items consumed by the users detected as similar to the target one. As was explained before, the grouping of users has been made through an FCA-based modelling, in the UserItemLattice.

The official results released by the ESWC 2014 Challenge organizers are shown in the Fig. 5 (ours identified by UNED). This figure shows the results obtained by all the participant groups (in the horizontal axis) after the evaluation period had finished, sorted by the F-Measure value (in the vertical axis).

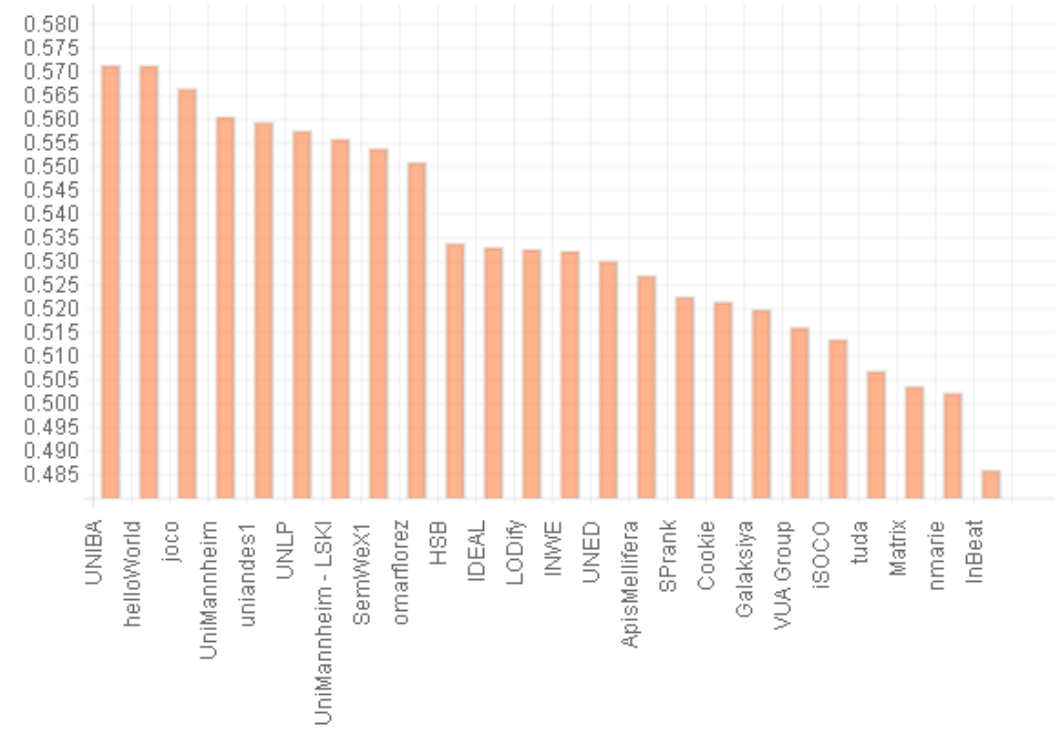

Fig. 5. ESWC2014 Interest Prediction Results 
As can be seen in the Fig. 5, our results are within the average range (note the bias in the values of the vertical axis); even though no LOD-based expansion technique was applied. Given these results, in the State-of-the-art of the task, it can be concluded that the performance of FCA to organize content can be applied in a recommendation scenario. And even more, the low performance obtained in the previous task does not seem to be attributable to FCA.

\section{Conclusions and Future work}

In this paper, a novel proposal based on Formal Concept Analysis has been presented to model user preferences by taking into account the LOD related to the items consumed by the users. More specifically, two modeling approaches were proposed, one based on a Collaborative-Filtering approach and one based on a Content-Based approach. A recommendation algorithm that improves the structured model provided by FCA to compute the recommendations was developed for each one.

To test the proposal the experimentation performed in the ESWC2014 Recommendation Challenge was presented. As a result of the analysis of the obtained results, it can be concluded that the FCA is a suitable technique to be applied in this kind of recommendation scenario.

The advantages of the inclusion of LOD in the proposed modelling it is still not clear. It seems that an accurate conceptual modelling based on LOD information could lead to better recommendations. However, the experimental results did not clearly confirm that point. In spite of this, no final conclusions can be drawn because of the general low performance.

At this point, two future lines of research appear. First, the LOD enrichment presented here is not as sophisticated as the top ones in the state of the art. So it has to be proved whether different management of the whole information in the LOD cloud could lead on a better performance approach. On the other hand, it seems that the ESWC 2014 scenario is especially challenging. In this sense, it could be that the application of the same approach in other recommendation scenarios may offer more representative results and more precise information.

As regards the FCA-related research, some open questions remain. An important FCA-related issue, barely coped on this paper, is how to take advantage of the "from generic to specific" structure of the obtained FCA-based model to adapt the recommendations. Another aspects not coped in this work for which FCA seems to be interesting are: the possibility of offering novel and unexpected recommendations based on the navigation through the lattice structure, or the trust-based implications derived from the possibility of showing the users information on the steps taken during the recommendations offered to them.

Acknowledgments. This work has been partially supported by the Regional Government of Madrid under Research Network MA2VIRMR (S2009/TIC-1542), and by the Spanish Government Project HOLOPEDIA (TIN 2010-21128-C02). 


\section{References}

1. Adomavicius, G., Tuzhilin, A.: Toward the next generation of recommender systems: A survey of the state-of-the-art and possible extensions. Knowledge and Data Engineering, IEEE Transactions on 17 (6), 734-749 (2005)

2. Ricci, F., Shapira, B.: Recommender systems handbook. Springer, Heidelberg (2011)

3. Brusilovsky, P., Chin, D.N.: Preface to the special issue on personalization in social web systems. User Model. User-Adapt. Interact. 23 (2-3), 83-87 (2013)

4. Vargas, S., Castells, P., Vallet, D.: Intent-oriented diversity in recommender systems. In: Proceedings of the 34th international ACM SIGIR conference on Research and development in Information Retrieval, pp. 1211-1212. ACM, New York, NY, USA (2011)

5. Pazzani, M.J., Billsus, D.: Content-based recommendation systems. In: The adaptive web. Springer, Heidelberg, 325-341 (2007)

6. Drucker, S.M., Fisher, D., Basu, S.: Helping users sort faster with adaptive machine learning recommendations. In: Human-Computer Interaction-INTERACT, 187-203 (2011)

7. Zhang, Y., Koren, J.: Efficient bayesian hierarchical user modeling for recommendation system. In: Proceedings of the 30th international ACM SIGIR conference on Research and development in information retrieval, pp. 47-54. ACM, New York, NY, USA (2007)

8. Schein, A.I., Popescul, A., Ungar, L.H., Pennock, D.M.: Methods and metrics for cold-start recommendations. In: Proceedings of the 25th international ACM SIGIR conference on Research and Development in Information Retrieval, pp. 253-260. ACM, New York, NY, USA (2002)

9. Boim, R., Milo, T., Novgorodov, S.: Diversification and refinement in collaborative filtering recommender. In: Proceedings of the 20th international ACM conference on Information and knowledge management, pp. 739-744. ACM, New York, NY, USA (2011)

10.Balabanovic, M., Shoham, Y.: Fab: content-based, collaborative recommendation. Communications of the ACM 40 (3), 66-72 (1997)

11.Bizer, C., Heath, T., Berners-Lee, T.: Linked data-the story so far. International journal on semantic web and information systems 5 (3), 1-22 (2009)

12.Passant, A.: dbrec - music recommendations using dbpedia. In Patel-Schneider, P., Pan, Y., Hitzler, P., Mika, P., Zhang, L., Pan, J., Horrocks, I., Glimm, B. (eds.) The Semantic Web: ISWC 2010. LNCS, vol. 6497, pp. 209-224. Springer Berlin Heidelberg (2010)

13.Heitmann, B., Hayes, C.: Using linked data to build open, collaborative recommender systems. In: AAAI Spring Symposium: Linked Data Meets Artificial Intelligence, pp. 76-81 (2010)

14.Di Noia, T., Mirizzi, R., Ostuni, V.C., Romito, D., Zanker, M.: Linked open data to support content-based recommender systems. In: Proceedings of the 8th International Conference on Semantic Systems, pp. 1-8. ACM, New York, NY, USA (2012)

15.Ostuni, V.C., Di Noia, T., Di Sciascio, E., Mirizzi, R.: Top-n recommendations from implicit feedback leveraging linked open data. In: Proceedings of the 7th ACM Conference on Recommender Systems, pp. 85-92. ACM, New York, NY, USA (2013)

16.Damljanovic, D., Stankovic, M., Laublet, P.: Linked data-based concept recommendation: Comparison of different methods in open innovation scenario. In Simperl, E., Cimiano, P., Polleres, A., Corcho, O., Presutti, V. (eds.) The Semantic Web: Research and Applications. LNCS, vol. 7595, pp. 24-38. Springer Berlin Heidelberg (2012)

17.Fernández, M., Cantador, I., López, V., Vallet, D., Castells, P., Motta, E.: Semantically enhanced information retrieval: An ontology-based approach. Web Semantics: Science, Services and Agents on the World Wide Web 9 (4), 434-452 (2011) 
18.Wille, R.: Concept lattices and conceptual knowledge systems. Computers \& mathematics with applications 23 (6), 493-515 (1992)

19.Ganter, B., Wille, R., Franzke, C.: Formal concept analysis: mathematical foundations. Springer-Verlag New York, Inc. (1997)

20.Simovici, D., Fomenky, P., Kunz, W.: Polarities, axiallities and marketability of items. In Cuzzocrea, A., Dayal, U. (eds.) Data Warehousing and Knowledge Discovery. LNCS, vol. 7448, pp. 243-252. Springer Berlin Heidelberg (2012)

21.Ignatov, D.I., Kuznetsov, S.O., Poelmans, J.: Concept-based biclustering for internet advertisement. In: Data Mining Workshop (ICDMW), IEEE 12th Int. Conf. pp. 123-130 (2012)

22.Senatore, S., Pasi, G.: Lattice navigation for collaborative filtering by means of (fuzzy) formal concept analysis. In: Proceedings of the 28th Annual ACM Symposium on Applied Computing. pp. 920-92. ACM, New York, NY, USA (2013)

23.Ignatov, D., Kaminskaya, A., Bezzubtseva, A., Konstantinov, A., Poelmans, J.: Fca-based models and a prototype data analysis system for crowdsourcing platforms. In Pfeiffer, H., Ignatov, D., Poelmans, J., Gadiraju, N. (eds.) Conceptual Structures for STEM Research and Education. LNCS, vol. 7735, pp. 173-192. Springer Berlin Heidelberg (2013)

24.Li, X., Murata, T.: A knowledge-based recommendation model utilizing formal concept analysis and association. In: Computer and Automation Engineering (ICCAE), 2010 The 2nd International Conference on. Volume 4. pp. 221-226 (2010)

25.du Boucher-Ryan, P., Bridge, D.: Collaborative recommending using formal concept analysis. Knowledge-Based Systems 19 (5), 309-315 (2006)

26.Khatchadourian, S., Consens, M.: Explod: Summary-based exploration of interlinking and rdf usage in the linked open data cloud. In Aroyo, L., Antoniou, G., Hyvnen, E., Teije, A., Stuckenschmidt, H., Cabral, L., Tudorache, T. (eds.) The Semantic Web: Research and Applications. LNCS, vol. 6089, pp. 272-287. Springer Berlin Heidelberg (2010) 